\title{
Spectrum Characteristics of Acoustic Emission Signals for Sandstone Instability
}

\author{
Y.B. Zhang ${ }^{1}$, X.H. Huang ${ }^{2, *}$ and B.Z. Tian ${ }^{1}$ \\ ${ }^{1}$ College of Mining Engineering, Hebei United University, Tangshan 063009, China \\ ${ }^{2}$ College of Information Engineering, Hebei United University, Tangshan 063009, China
}

Received 5 April 2014; Accepted 10 September 2014

\begin{abstract}
In order to predict the rock rupture and instability, acoustic emission signals of natural sandstones and hydrous sandstones are analyzed through the Fast Fourier Transform and the Welch method. It is found that the acoustic emission signals of the hydrous sandstones have narrower range of frequency spectrum and lower average dominant frequencies than the natural sandstones. The dominant frequencies of the acoustic emission signals of the hydrous sandstones reduce more dramatically. In addition, two types of Welch power spectrum of the acoustic emission signals in terms of the shape are presented in this paper, which are named Type A and Type B. The Type B Welch power spectrum emerges when the acoustic emission occurs frequently, in which a disaster tends to appear. Time baselines are selected when relative stronger cracks and crack coalescence happens. We find that the greater signals energy at the time baseline, the higher probability of occurrence of Type B spectrum appears before the time baseline. Also, the probabilities of Type B before the corresponding the baseline time of rock last rupture in hydrous sandstones are higher than that of natural sandstones.
\end{abstract}

Keywords: Acoustic Emission, Fast Fourier Transform, Welch Power Spectrum, Rock Rupture, Sandstone

\section{Introduction}

The frequency characters of Acoustic Emission (AE) have been studied by some experts, and some results have been obtained about dry rock samples with loading [1-5]. Y.P. Chugh presents that the spectrum character is expressed by two main frequency bands which are $0 \mathrm{kHz}-6 \mathrm{kHz}$ and 10 $\mathrm{kHz}-15 \mathrm{kHz}$, or is expressed by main energy band [6]. While R.M. Koerner finds that the main frequency interval is between $0 \mathrm{kHz}-50 \mathrm{kHz}$, and it has the trend of shifting to the low frequency with adding stress [7]. Cai analyzes that main frequency bands are $200 \mathrm{kHz} 2000 \mathrm{kHz}$ [8]. K. Zhao's results show that the secondary-dominant frequency of $\mathrm{AE}$ arises in all cycles, and frequency range of $\mathrm{AE}$ was wider with the increasing of the stress level [9]. In 1995, it had been found by Martyn that waves with low amplitude and high frequency were primary waves before frequency peak value came, and low frequency signals were major ones after stress peak value about sandstones [10]. A few researchers find that the AE characteristics of dry rocks and hydrous rocks are significantly different when rocks are under seepage action [11-12].

However, it has not been given enough attention to the research on the peak frequency value regular and average peak frequency of $\mathrm{AE}$ in the above mentioned references. And the systematic and in-depth researches are required to observe the AE characteristics of rock failure such as, the AE occurrence process, the development and the abnormal phenomenon. This paper presents the analysis of the AE signals of natural sandstones and hydrous sandstones from two perspectives.

\footnotetext{
*E-mail address: txhxh@163.com

ISSN: 1791-2377 @ 2014 Kavala Institute of Technology. All rights reserved.
}

In this study, FFT and Welch spectrum analysis to rock AE signals is used to predict the rock rupture and instability. A variety of experiments are conducted in the twenty selected sandstones which are divided into two groups in terms of humidity. Using FFT algorithm, the frequency spectrum ranges and the shifting pattern of the dominant frequency of the $\mathrm{AE}$ signals are analyzed to obtain an appropriate band for monitoring $\mathrm{AE}$ signals during rock rupture. The study endeavors to create a baseline of determining the monitoring parameters in the prediction of a disaster during the mining procedure.

Experimental configurations are provided in section 2 . Section 3 illustrates the FFT spectrum analysis of the AE signals while Section 4 describes the Welch spectrum analysis of AE signals. Section 5 functions as the conclusion of the paper.

\section{Experimental Configurations}

The test objects are sandstones. The dominant frequency of sandstone is studied through the indoor rock mechanics test. Laboratory experiments are operating in accordance with the relevant regulations. Firstly, Experimental rock samples are taken from the actual mining rock, which can representatively be used as the carrier to study rock properties. Secondly, the test pieces were processed into standard rock samples of $50 \mathrm{~mm} \times 50 \mathrm{~mm} \times 100 \mathrm{~mm}$ based on rock mechanics experimental protocols. The sandstones are divided into two groups: the natural group and the hydrous group. The hydrous group of sandstones is moisturized by way of the freedom absorbent method to saturate them in the water for 48 hours. The natural group is not moisturized. 
The experimental configurations of the two groups are the same. The testing machine uses displacement loading mode with the loading rate of $0.03 \mathrm{~mm} / \mathrm{min}$. The uniaxial compression is used to conduct the experiments. The threshold value of the AE instrument is $40 \mathrm{~dB}$. The sampling points are 1024 and the sampling rate is $1 \mathrm{MHz}$.

We take two sandstones (one named SY-3 belongs to natural group, another named HSSY-3 belongs to hydrous group) as examples to draw the curves of $\mathrm{AE}$ events energy/time which are demonstrated in Fig. 1. The largest energy time of SY-3 and HSY-3 are $1962.34715 \mathrm{~s}$ and $558.2327 \mathrm{~s}$ respectively, which is corresponding to the time of releasing powerful energy resulted from high rupture. The following analysis is based on the acoustic emission signals before this time base-line.

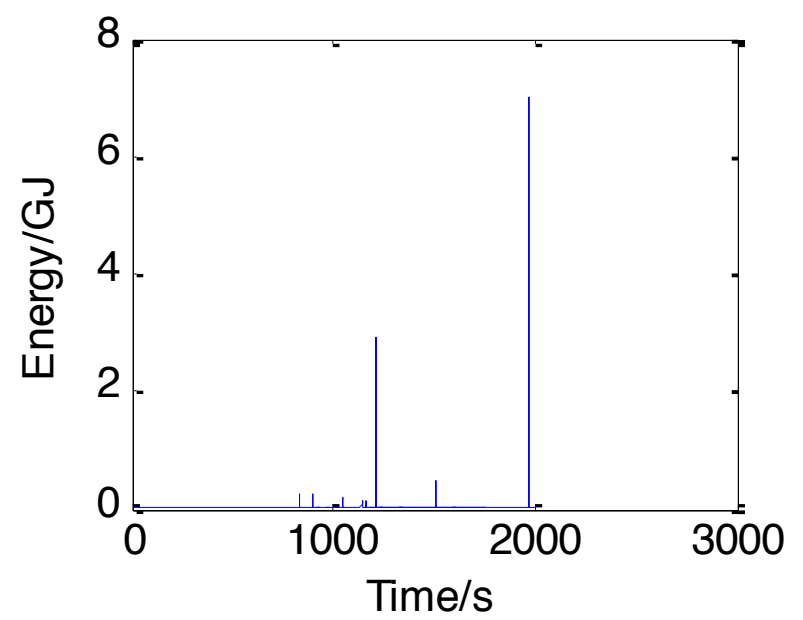

(a) AE events energy/time for natural sandstone

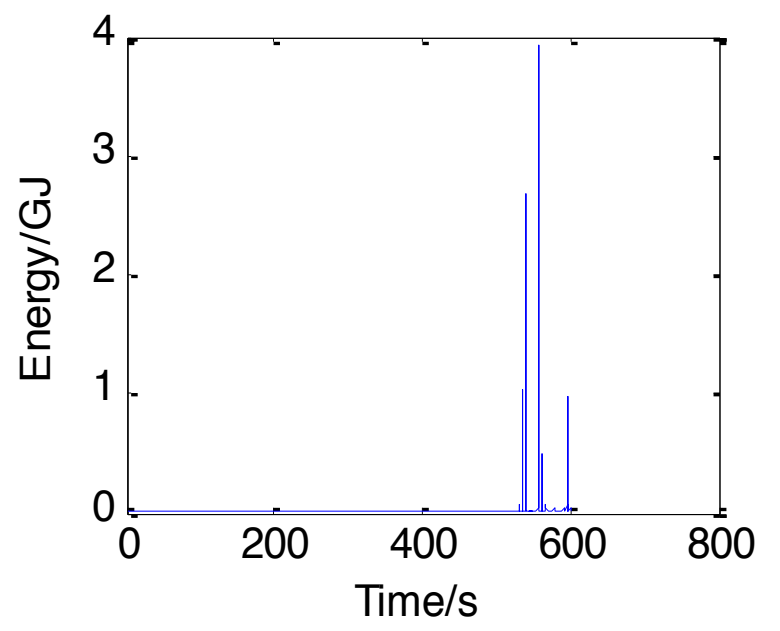

(b) AE events energy/time for hydrous sandstone

Fig.1. Curves of AE events energy/time

\section{FFT Spectrum Analysis of Acoustic Emission}

The spectrum analysis is a general method to study the waveform analysis of the AE signals. The AE features can be emphasized by analyzing the distribution characteristics of the AE source signals in the frequency domain. In this study, the frequency spectrum ranges and the shifting trends of the dominant frequency of the AE signals of the natural group and the hydrous group respectively are analyzed. To reflect the internal fracture of the rock accurately, wavelet transform is performed on the acoustic emission signals before doing the spectrum analysis, which is a good method to remove the mixed noise from the AE signals [13-15].
For the natural group, the SY-3 sample in the natural state is randomly selected. 450 sets of AE signals in a chronological order are analyzed. The dominant frequencies of these signals are calculated by FFT algorithm. Fig. 2(a) shows the dominant frequencies, in which the horizontal axis represents the 450 sets of $\mathrm{AE}$ signals and the vertical distribution within the range from $35 \mathrm{kHz}$ to $65 \mathrm{kHz}$ in the axis represents the dominant frequencies. The dominant frequencies of $\mathrm{AE}$ signals of sandstone mainly concern natural group (Fig. 2(a)). In addition, the dominant frequencies tend to gradually decrease while the loading increases. The mean value of these dominant frequencies of the 450 sets of signals was $42.72 \mathrm{kHz}$.

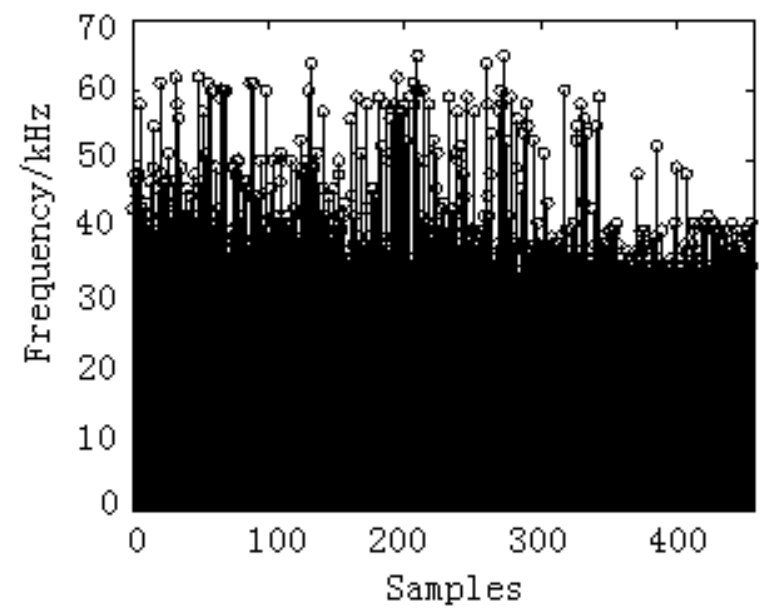

(a) Dominant frequencies of the natural group

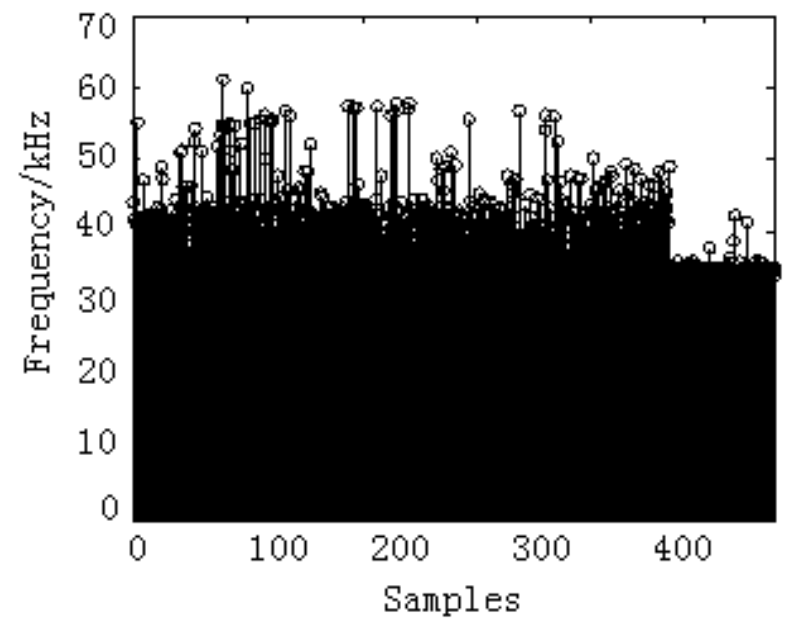

(b) Dominant frequencies of the hydrous group

Fig.2. Dominant frequencies of the acoustic emission signals

The HSSY samples are also randomly selected for the experiments of the hydrous group. The AE signals are observed in the same way as what have been done in the natural group. The dominant frequencies of AE signals are computed using FFT algorithm and the results are shown in Fig. 2(b). The dominant frequencies of the AE signals of the hydrous sandstones were mainly distributed within the range from $35 \mathrm{kHz}$ to $60 \mathrm{kHz}$ and the dominant frequencies generally decreased with the load increasing (Fig. 2(b)). In addition, the mean value of these dominant frequencies was $41.7770 \mathrm{kHz}$.

Further experiments are conducted in the natural group (named SY) and hydrous group (named HSSY) respectively. Six sandstones are selected for each group. The frequency spectrum analysis results of two groups are listed in Table 1. 
The results showed the same conclusion was drawn as the former experiments. In other words, the frequency spectrums of the AE signals of both the natural group and the hydrous group are more intensive when $\mathrm{AE}$ events occur more frequently, which means that the power is more concentrated. In addition, the dominant frequencies of $\mathrm{AE}$ signals have the trend of gradually decreasing as load increases.

The differences between the natural group and the hydrous group are also identified. The AE signals of former specimens have wider spectral ranges than those of the latter specimens. Moreover, when releasing energy during rock rupture the frequency spectrums are more concentrated in experiments of the former specimens. The mean value of dominant frequencies of the hydrous group is $1 \mathrm{KHz}$ which is lower than that in the natural group. That is because the threshold values of rock subcritical crack growth were reduced by water. Water speeds up the subcritical crack growth, which implies that the subcritical crack growth velocity of saturated water specimen is speeded at same stress intensity factor of air specimens, which implies that water speeds up the subcritical crack growth. The microfracture of water-saturated rock produced faster in the subcritical state, which lead to high-frequency absorption increase, so the frequency declined.

\section{Welch Spectrum Analysis of Acoustic Emission}

Power spectrum analysis technology is very important to signal analysis and recognition. Welch method is an improved and effective method of the power spectrum analysis [16-18]. This method can lower spectrum estimation variance without resolution reduction by splitting signals into overlapping segments and adding window.

In this method, the original data segment $x(n)$ of length $N$ is split up into $p$ data segments of length $M$, then the $p-t h$ improved periodogram is listed as follows,

$J_{P}(\omega)=\frac{1}{M U}\left|\sum_{n=0}^{M-1} x_{p}(n) w(n) e^{-j \omega n}\right|^{2}$

Where $U(\omega)=\frac{1}{M} \sum_{n=0}^{M-1} w^{2}(n)$ is the normalization factor, $w(n)$

is window function. The power spectrum estimate of the whole signal $x(n)$ can be obtained by doing time average to all individual periodograms, that is

$$
\hat{B}_{\omega}(\omega)=\frac{1}{p} \sum_{p=0}^{p} J_{p}(\omega)
$$

Welch method is used in this paper to provide the energy transformation of AE signals during the process of rock rupture, which reveals the rock failure mechanism. Both the instability of natural group and hydrous group acoustic emission signals during the rupture process are analyzed, in which ten sandstones are selected for each group. We find a common phenomenon in the two groups: the power spectrum of the AE signals has two primary shapes. The two shape as Type A (Fig. 3(a)) and Type B (Fig. 3(b)) are defined. The corresponding phase shape are displayed in Fig. 4(a) and Fig. 4(b). Seen from Fig. 4, the phase of Type A is linear during some frequency ranges, while the phase of Type B has a severe discontinuous characteristic.
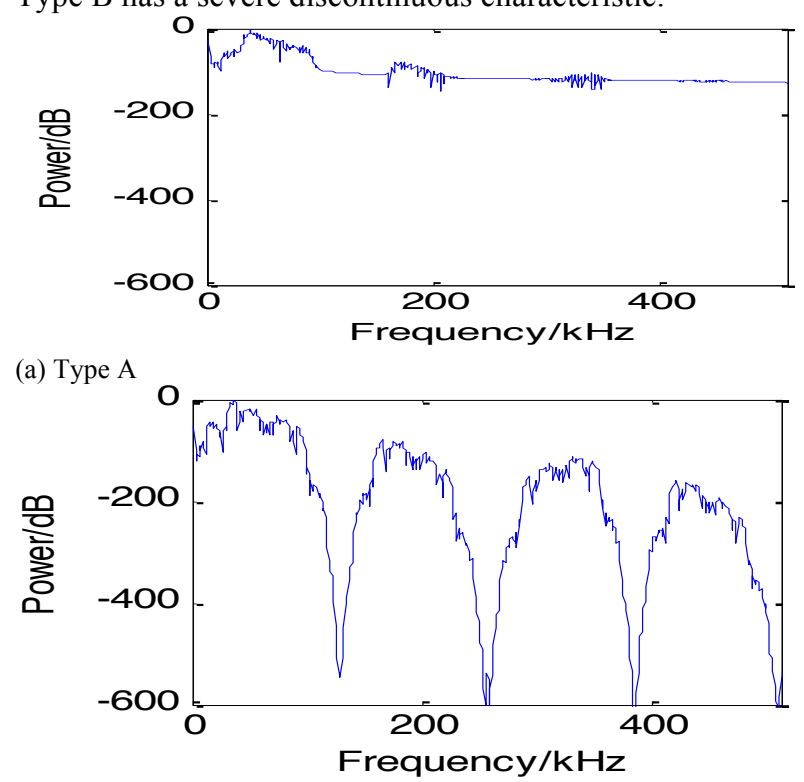

(b) Type B

Fig.3. Two kinds of Welch power spectrum shape during the loading process

Table 1. Frequency spectrum characteristics of AE signals of sandstone in two groups

\begin{tabular}{c|c|c|c}
\hline Sandstone No. & $\begin{array}{c}\text { Frequency range } \\
\text { (kHz) }\end{array}$ & $\begin{array}{c}\text { Dominant frequency } \\
\text { (kHz) }\end{array}$ & $\begin{array}{c}\text { Average frequency } \\
(\mathbf{k H z})\end{array}$ \\
\hline SY-1 & $10 \sim 102$ & $35 \sim 64$ & 42.5104 \\
SY-2 & $11 \sim 99$ & $34 \sim 66$ & 42.7002 \\
SY-3 & $10 \sim 100$ & $35 \sim 65$ & 42.7200 \\
SY-4 & $10 \sim 99$ & $34 \sim 64$ & 42.6124 \\
SY-5 & $9 \sim 100$ & $36 \sim 66$ & 42.7244 \\
SY-6 & $10 \sim 100$ & $35 \sim 66$ & 42.7210 \\
HSSY-1 & $10 \sim 94$ & $33 \sim 60$ & 41.6012 \\
HSSY-2 & $11 \sim 94$ & $32 \sim 59$ & 41.5721 \\
HSSY-3 & $10 \sim 95$ & $35 \sim 60$ & 41.7653 \\
HSSY-4 & $9 \sim 94$ & $34 \sim 60$ & 41.6910 \\
HSSY-5 & $10 \sim 94$ & $35 \sim 59$ & 41.6985 \\
HSSY-6 & $10 \sim 95$ & $35 \sim 60$ & 41.7770 \\
\hline
\end{tabular}

The 23 events signals (No.129836, No.129837 No.129838, ‥, No.129857 and No.129858) were chosen during $0.434 \mathrm{~s}$ of one nature sandstone (SY-3) to do phase spectrum analysis, then Fig.5 is obtained. The last subgraph in the Fig.5 is the phase spectrum of the largest energy event (No.129858). The phase of the largest energy event is Type
$\mathrm{A}$, and there emerges Type B phase before the crack is coming.

A lot of experiments have been done. Two conclusions can be drawn:

(1) Type A Welch power spectrum dominates when the AE occurs infrequently, the Type B Welch power spectrum 
emerges besides Type A spectrum when the AE occurs frequently, in which the original cracks continue expanding while new cracks occur, then new cracks and the existing cracks coalesce in the sandstones.

(2) In addition, the Type B Welch power spectrum is mainly observed before the emergence of $\mathrm{AE}$ signals with large energy when the sandstones crack relatively strongly and/or the cracks coalesce.

Five groups of AE signals that have great energy as time baselines from the experiments of the two groups are

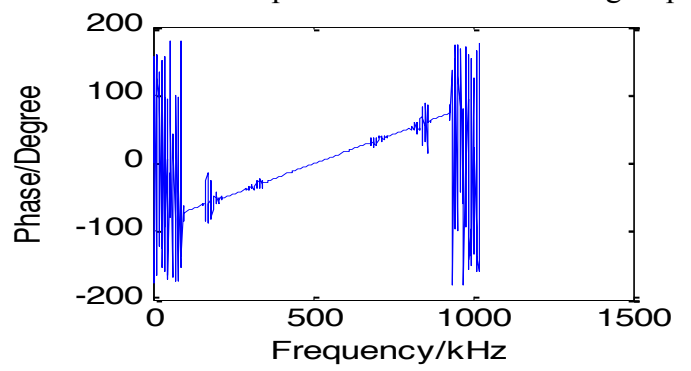

(a) Type A

Fig.4. Two kinds of Phase spectrum during the loading process selected. It is the time when relatively stronger cracks and crack coalescence happens. 200 sets of signals before the time baseline are analyzed to identify the occurrence probability of the Type B power spectrum before the strong cracks happen when a disaster probably emerges. Table 2 and Table 3 show the results of the natural group and the hydrous group respectively.

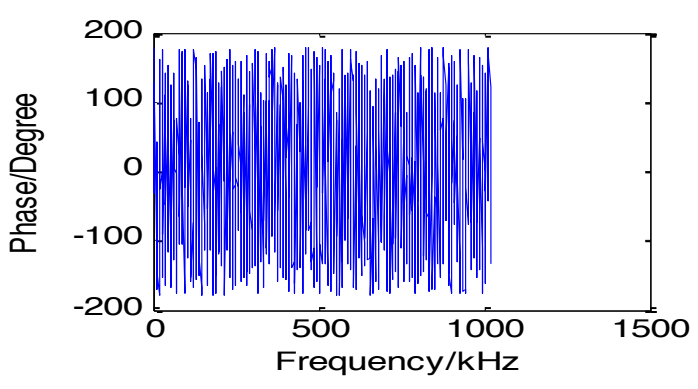

(b) Type B

Table 2. Occurrence probability of Type B power spectrum before the time baseline of the natural group

\begin{tabular}{lccccc}
\hline Signals with larger energy & No.13023 & No. 17883 & No. 54477 & No. 85141 & No. 129858 \\
\hline Time value/s & 277.2419 & 299.0921 & 402.2333 & 501.0066 & 654.1157 \\
\hline Energy value/J & $2.52 \mathrm{E}+08$ & $2.59 \mathrm{E}+08$ & $2.92 \mathrm{E}+09$ & $4.78 \mathrm{E}+08$ & $6.99 \mathrm{E}+09$ \\
\hline Probability of type B power spectrum & $10.5 \%$ & $13 \%$ & $33 \%$ & $21 \%$ & $44 \%$ \\
\hline
\end{tabular}

Table 3. Occurrence probability of Type B power spectrum before the time baseline of the hydrous group

\begin{tabular}{lccccc}
\hline Signals with larger energy & No.38193 & No.41566 & No.52190 & No.54533 & No.75125 \\
\hline Time value/s & 534.1603 & 539.9449 & 558.2327 & 563.0505 & 597.2053 \\
\hline Energy value/J & $1.03 \mathrm{E}+09$ & $2.68 \mathrm{E}+09$ & $3.92 \mathrm{E}+09$ & $4.89 \mathrm{E}+08$ & $9.68 \mathrm{E}+08$ \\
\hline Probability of type B power spectrum & $31 \%$ & $42 \%$ & $57.5 \%$ & $13.5 \%$ & $20 \%$ \\
\hline
\end{tabular}
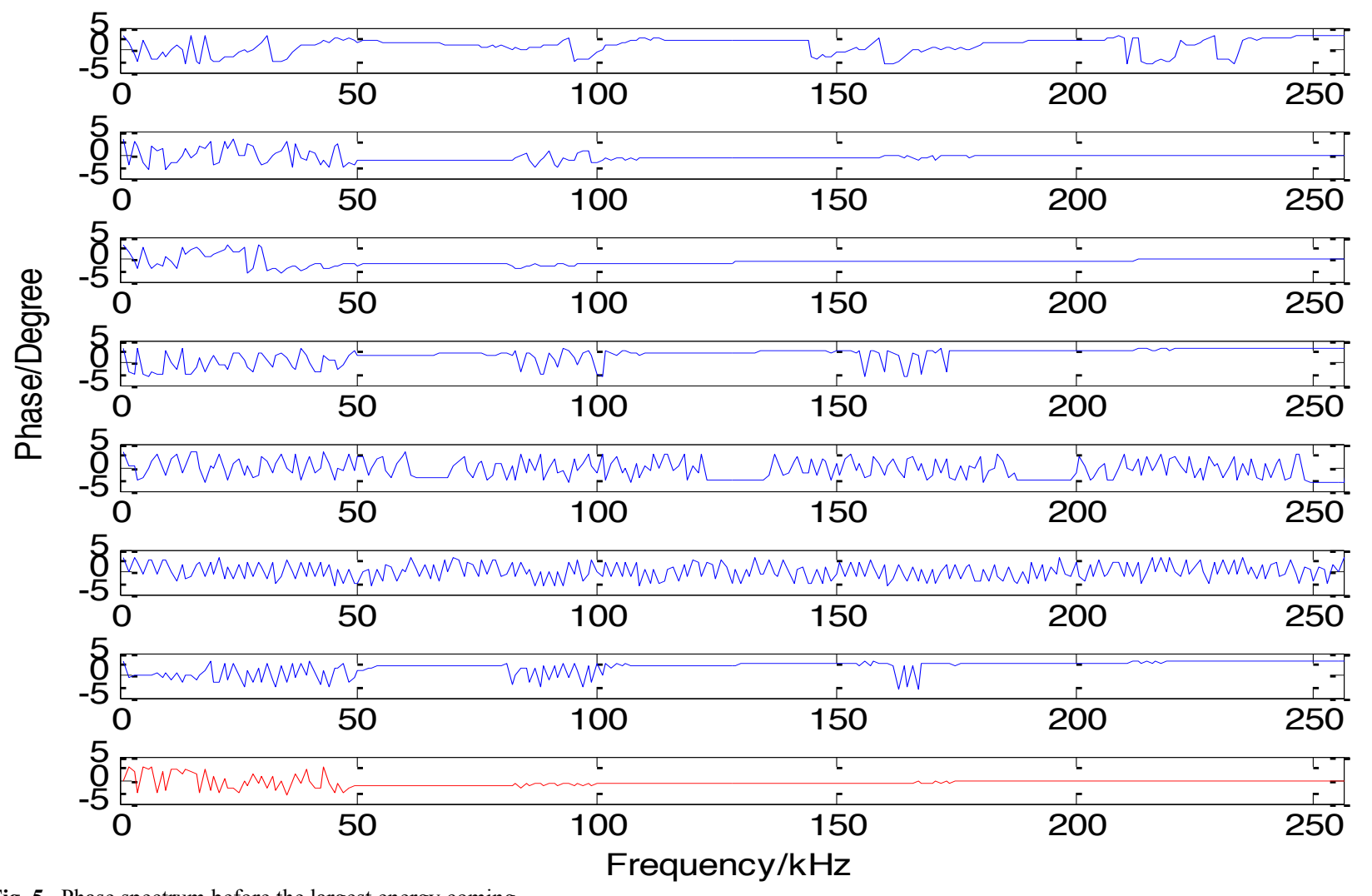

Fig. 5. Phase spectrum before the largest energy coming 
In the natural group, the AE signal in the point of No.129858 was the largest energy signal $(6.99 \mathrm{E}+09)$ when the specimen macroscopically ruptures (Tab.2). The probability of Type B power spectrum occurrence in the selected 200 sets of signals before this time was as high as $44 \%$. The second largest-energy was in the point of No.54477 with the energy of 2.92E+09 (Tab.2), when the probability in the selected 200 sets of signals was $33 \%$. Table 3 shows the probability of Type B power spectrum occurrence in the selected 200 sets of signals before the corresponding time baseline in the hydrous group. The result is that the signal with largest energy $(3.92 \mathrm{E}+09)$ was at the point of No.41566 when the largest corresponding probability was indentified $(57.5 \%)$. By contrast, the least probability was $13.5 \%$ before the time baseline when the signal energy had the lowest value $(4.89 \mathrm{E}+08)$.

\section{Conclusion}

In this study, FFT and Welch spectrum analysis to sandstone acoustic emission signal are applied to predict the rock rupture and instability. A variety of experiments are conducted in the twenty selected sandstones, which are divided into two groups: the natural group and the hydrous group. The conclusion is that the spectrum range of the acoustic emission signals in the hydrous group is slightly narrower than those in the natural group. The average value of the dominant frequencies of the AE signals in the hydrous group is $1 \mathrm{kHz}$ which is lower than that in the natural group.

From the Welch spectrum analysis, two types of spectrum are identified: Type A and Type B. The Type B Welch power spectrum emerges besides Type A spectrum when the acoustic emission occurs frequently, in which the original cracks continue expanding, new cracks occur, and then the new cracks and the existing cracks coalesce in the sandstones. Moreover, the greater signals energy at the time baseline, the higher possibility of occurrence of Type B spectrum before the time baseline. Also, the closer to the time baseline, the higher possibility of occurrence of Type B spectrum was. In addition, the water affects acoustic emission signals of the sandstones. The probability of Type B power spectrum occurrence before the time baseline of last rupture of the rock is different in the two groups.

In our experiments, several hundreds of data in accordance with the time are computed, and time baselines are selected when relatively stronger cracks and crack coalescence happens, although it can reflect the rules of the frequency changing, a software should be programmed in the future, so that we can draw the frequency peak values during the whole process and get the trend of the spectrum change according to the load. And some quantity characteristics of the Welch spectrum should be explored so that the clue of the rock fracture can be clearly obtained. Also more experiments should be done to test the results.

\section{Acknowledgments}

This work was financially supported by the National Natural Science Foundation of China (No.51174071), and the Hebei Province Natural Science Fund (E2012209047). All these are gratefully acknowledged.

\section{References}

[1]. Brouillard T.-F.: "Introduction to Acoustic emission", Materials Evaluation, 46 (7), 1988, pp. 174-180.

[2]. Ravindra H.-V., "Some aspects of acoustic emission signal processing", Journal of Materials Processing Technology, 109, 2001, pp. 242-247.

[3]. Tham L.-G., Liu H., Tang C.-A., Lee P.-K. and Tsui Y., "On tension failure of $2 \mathrm{D}$ rock specimens and associated acoustic emission", Rock Mechanics and Rock Engineering, 38 (1), 2005, pp. 1-19.

[4]. Prikry L.-R., Lokajicek T., Li C. and Rudajev V., "Acoustic emission characteristics and failure of uniaxially stressed granitic rocks: The effect of rock fabric", Rock Mechanics and Rock Engineering, 36 (4), 2003, pp. 255-270.

[5]. Rudajev V., Vilhelm J. and Lokajicek T., "Laboratory studies of acoustic emission prior to uniaxial compressive rock failure", Int J Rock Mech. \& Min. Sci, 37 (4), 2000, pp. 699-704.

[6]. Chugh Y.-P. and Stefanko R., "Investigation of the frequency spectrum of microseismic activity in rock under tension", The 10th U.S. Symposium on Rock Mechanics (USRMS), 20-22 May, Austin, Texas, 1968, pp. 42.

[7]. Koerner R.-M. and Lord A.-E., "AE detection of prestress in soil and rock", Proceedings of the Fourth Conference on AE/MA in Geologic structures and materials, Pennsylvania, U.S.A., 1989, pp. 73-86.

[8]. Cai M., Kaiser P.-K., Morioka H., Minami M., Maejima T., Tasaka Y. and Kurose H., "FLAC/PFC coupled numerical simulation of $\mathrm{AE}$ in large-scale underground excavations", International Journal of Rock Mechanics and Mining Science, 44, 2007, pp. 550-564.

[9]. Zhao K., Lin Y. and Xing X., "Studies on Acoustic Emission Frequency of Creep of Sandstone under Different Stress Levels", Copper Engineering, 4, 2012, pp. 22-24.
[10]. Read M.-D., Ayling M.-R., Mereditth P.-G. and Murrell S.-A., "Micro cracking during triaxial deformation of porous rocks monitored by changes in rock physical properties (II)-Pore volumometry and acoustic emission measurements on watersaturated rocks", Technology physics, 245, 1995, pp. 223-235.

[11]. Brian J.-M., "Seepage properties of a single rock fracture subjected to triaxial stresses", Natural Science, 17 (12), 2007, pp. $1482-1486$.

[12]. Liu C., Chen C. and Fu S., "Testing study on seepage characteristics of a single fracture with sand under shearing displacement", Chinese Journal of Rock Mechanics and Engineering, 21 (10), 2002, pp. 1457-1461. (in Chinese)

[13]. Xie H., Pierce L.-E., Ulaby F.-T., "SAR speckle reduction using wavelet denoising and Markov random field modeling", Geoscience and Remote Sensing, IEEE Transactions on, 40 (10), 2002, pp. 2196-2212.

[14]. Cho D.-W., Bui T.-D. and Chen G.-Y., "Multiwavelet statistical modeling for image denoising using wavelet transforms", Signal Processing: Image Communication, 20 (1), 2005, pp. 77-89.

[15]. Liu L., Li S., Zhang H., Zhang C. and Jia Z., "Eigenfaces and identification of colour face images", Computer Modelling and New Technologies, 17 (4), 2013, pp. 136-141.

[16]. O'Leary B. and Kelley J.-A., "Utilization of the coherence function with Welch's method for signal analysis in low resolution laser-induced breakdown spectroscopy", Society for Applied Spectroscopy, 64 (4), 2010, pp. 370-376.

[17]. Luo M., and Liu S., "Analysis of Welch method in PSE based on matlab", Journal of Beijing technology and business university (Natural Science Edition), 25 (3), 2007, pp. 58-60. (in Chinese)

Ahmet A. and Ahmet S.-Y., "Frequency domain analysis of power system transients using Welch and Yule-Walker AR methods", Energy Conversion and Management, 48 (7), 2007, pp. 21292135. 